Corrigendum

\title{
Corrigendum to "miR-221 Alleviates the Ox-LDL-Induced Macrophage Inflammatory Response via the Inhibition of DNMT3b-Mediated NCoR Promoter Methylation"
}

\author{
Jinshan Ye, ${ }^{1,2}$ Yaxi Wu, ${ }^{3}$ Ruiwei Guo $\mathbb{D},{ }^{1}$ Wenjun Zeng, ${ }^{1}$ Yanan Duan, ${ }^{1}$ Zhihua Yang, \\ and Lixia Yang $\mathbb{D}^{1}$ \\ ${ }^{1}$ Department of Cardiology, 920th Hospital of PLA Joint Logistic Support Force, Yunnan 650032, China \\ ${ }^{2}$ Department of Cardiology, Tongren Hospital, Yunnan 650032, China \\ ${ }^{3}$ Institution of Cardiovascular Research, Xinqiao Hospital, Third Military Medical University, Chongqing 400037, China \\ Correspondence should be addressed to Lixia Yang; doctorylixia@aliyun.com
}

Received 24 February 2020; Accepted 25 March 2020; Published 21 August 2020

Copyright (c) 2020 Jinshan Ye et al. This is an open access article distributed under the Creative Commons Attribution License, which permits unrestricted use, distribution, and reproduction in any medium, provided the original work is properly cited.

In the article titled "miR-221 Alleviates the Ox-LDL-Induced Macrophage Inflammatory Response via the Inhibition of DNMT3b-Mediated NCoR Promoter Methylation"[1], the authors Wenjun Zeng, Yanan Duan, and Zhihua Yang were incorrectly affiliated to the institution "Department of Cardiology, Tongren Hospital, Yunnan 650032, China." The correct affiliation for these authors is "Department of Cardiology, 920th Hospital of PLA Joint Logistic Support Force, Yunnan 650032, China."

The corrected affiliations are shown in the author information above.

\section{References}

[1] J. Ye, Y. Wu, R. Guo et al., "miR-221 alleviates the Ox-LDLinduced macrophage inflammatory response via the inhibition of DNMT3b-mediated NCoR promoter methylation," Mediators of Inflammation, vol. 2019, Article ID 4530534, 15 pages, 2019. 